Martin Obermeier, Monia Pacenti, Robert Ehret, Francesco Onelia, Rory Gunson, Emily Goldstein, Stéphane Chevaliez, Alba Vilas, Allison Glass, Leana Maree, Maria Krügel, Heribert Knechten, Patrick Braun, Gudrun Naeth, Francesca Azzato, Danijela Lucic, Natalia Marlowe, Michael John Palm, Karin Pfeifer, Birgit Reinhardt, Jens Dhein, Ajith Mathew Joseph, Laura Martínez-García and Juan-Carlos Galán*

\title{
Improved molecular laboratory productivity by consolidation of testing on the new random-access analyzer Alinity $\mathbf{m}$
}

https://doi.org/10.1515/labmed-2020-0102

Received August 28, 2020; accepted October 15, 2020;

published online November 9, 2020

\section{Abstract}

Objectives: Automated molecular analyzers have accelerated diagnosis, allowing earlier intervention and better

Martin Obermeier and Monia Pacenti contributed equally to this work.

*Corresponding author: Juan-Carlos Galán, Servicio de Microbiología, Hospital Universitario Ramón y Cajal and Instituto Ramón y Cajal de Investigación Sanitaria (IRYCIS), CIBER en Epidemiología y Salud Pública (CIBERESP), Crta de Colmenar Km 9,1, 28034, Madrid, Spain, Phone: +34 91 3368787, E-mail: juancarlos.galan@salud.madrid.org Martin Obermeier and Robert Ehret, Medizinisches

Infektiologiezentrum, Berlin, Germany

Monia Pacenti, Azienda Ospedaliera di Padova, Padua, Italy

Francesco Onelia, University of Padua, Padua, Italy

Rory Gunson and Emily Goldstein, West of Scotland Specialist

Virology Centre, Glasgow, UK

Stéphane Chevaliez, Hôpital Universitaire Henri Mondor, Créteil, France

Alba Vilas, Laboratori de Referència de Catalunya, El Prat de Llobregat, Spain

Allison Glass, Leana Maree and Maria Krügel, Lancet Laboratories, Johannesburg, South Africa

Heribert Knechten, Patrick Braun and Gudrun Naeth, PZB Aachen,

Aachen, Germany

Francesca Azzato, Victorian Infectious Diseases Reference

Laboratory, Melbourne, Australia

Danijela Lucic, Natalia Marlowe, Michael John Palm and Ajith Mathew Joseph, Abbott Molecular Inc., Des Plaines, IL, USA. https://orcid.org/ 0000-0003-2001-9465 (A.M. Joseph)

Karin Pfeifer, Birgit Reinhardt and Jens Dhein, Abbott GmbH, Wiesbaden, Germany. https://orcid.org/0000-0002-7200-2967 (J. Dhein)

Laura Martínez-García, Servicio de Microbiología, Hospital Universitario Ramón y Cajal and Instituto Ramón y Cajal de Investigación Sanitaria (IRYCIS), CIBER en Epidemiología y Salud Pública (CIBERESP), Madrid, Spain patient follow-up. A recently developed completely automated molecular analyzer, Alinity ${ }^{\mathrm{TM}} \mathrm{m}$ (Abbott), offers consolidated, continuous, and random-access testing that may improve molecular laboratory workflow.

Methods: An international, multicenter study compared laboratory workflow metrics across various routine analyzers and Alinity $m$ utilizing assays for human immunodeficiency virus type 1 (HIV-1), hepatitis C virus (HCV), hepatitis $\mathrm{B}$ virus (HBV), high-risk human papillomavirus (HR HPV), and sexually transmitted infection (STI) (Chlamydia trachomatis [CT]/Neisseria gonorrhoeae [NG]/Trichomonas vaginalis [TV]/Mycoplasma genitalium [MG]). Three turnaround times (TATs) were assessed: total TAT (sample arrival to result), sample onboard TAT (sample loading and test starting to result), and processing TAT (sample aspiration to result).

Results: Total TAT was reduced from days with routine analyzers to hours with Alinity $\mathrm{m}$, independent of requested assays. Sample onboard TATs for standard workflow using routine analyzers ranged from 7 to $32.5 \mathrm{~h}$ compared to 2.75-6 $\mathrm{h}$ for Alinity $\mathrm{m}$. The mean sample onboard TAT for STAT samples on Alinity m was $2.36 \mathrm{~h}( \pm 0.19 \mathrm{~h})$. Processing TATs for Alinity $m$ were independent of the combination of assays, with $100 \%$ of results reported within $117 \mathrm{~min}$.

Conclusions: The consolidated, continuous, randomaccess workflow of Alinity $m$ reduces TATs across various assays and is expected to improve both laboratory operational efficiency and patient care.

Keywords: diagnosis; lab automation; molecular assay; turnaround time; workflow.

\section{Introduction}

In the era of personalized medicine and targeted treatments, clinical decision-making and patient care rely on rapid access to accurate diagnostic test results. 
Development of nucleic acid amplification tests (NAATs) has improved the diagnosis of viral diseases. Although this technological revolution in molecular diagnostics was initially restricted to virology, such as for human immunodeficiency virus type 1 (HIV-1), hepatitis C virus (HCV), hepatitis $B$ virus (HBV), or high-risk human papillomavirus (HR HPV), NAATs have since been applied to bacteriology, for the diagnosis of sexually transmitted infections (STIs), including Chlamydia trachomatis (CT) and Neisseria gonorrhoeae (NG). These examples represent infections with high disease burden requiring the implementation of automated platforms [1, 2]. Consequently, clinical laboratories have seen consolidation of various testing departments into the molecular diagnostic space in a continuous process of technological evolution [3].

Yet these diagnostic advances harbor new challenges, including the pressure to report results from multiple tests faster, often on multiple specimen types. As the application of molecular testing has expanded, it is now recognized that rapid generation of molecular test results could lead to earlier diagnosis and treatment, which may significantly improve patient outcomes [4]. With the adoption of national screening programs (e.g., for CT, NG, and molecular detection of HR HPV DNA in cervical cancer screening) and large-scale diagnostic testing in response to pandemics, laboratories are being tasked with testing large volumes of samples on abbreviated time scales to prevent disease transmission and improve public health [5, 6].

These challenges have motivated clinical laboratories to adopt high-level automation to cope with the new demands [7-9], including consolidating menus on fewer platforms and achieving shorter turnaround times (TATs) [10]. The implementation of automated molecular analyzers is allowing fundamental shifts in the workflow within the clinical laboratory, for example, moving from batch testing to continuous processing of samples. To address the growing challenges and demands on the modern molecular laboratory, and in response to feedback from the clinical community, Abbott Molecular (Des Plaines, USA) developed a polyvalent automated molecular platform, Alinity ${ }^{\mathrm{TM}} \mathrm{m}$ (CE, IVD) [11].

Alinity $m$ differs from other molecular diagnostic systems in that samples can be tested as soon as they arrive in the molecular laboratory using a streamlined workflow combined with rapid result reporting. The purpose of the present analysis was to evaluate the impact on workflow of consolidation on a single platform vs. testing on multiple platforms. The study examined the time from initial sample receipt and processing (pre-analytics) to result reporting (post-analytics) [12] at eight independent molecular diagnostic laboratories in routine clinical settings.

\section{Materials and methods}

\section{Study design}

This international, multicenter study compared the workflows, comprising receipt, preparation, and analysis of samples for HIV-1, HCV, HBV, HR HPV, and STI (CT, NG, Trichomonas vaginalis [TV], and Mycoplasma genitalium [MG]) assay analytes, performed on Alinity $\mathrm{m}$ and various analyzers widely used in clinical laboratories.

The study utilized either simulated specimens (six study sites) or residual de-identified patient specimens (two study sites) using the respective laboratory's sample arrival pattern for testing on Alinity m. Clinical samples for routine testing consisted of serum, plasma, liquidbased cytology medium, urine, and various swab specimens, including oropharyngeal, vaginal, endocervical, urethral, and rectal swabs. Residual patient specimens (where applicable) were sourced per site policies, procedures, and applicable local regulations, including approval by the site's Independent Ethics Committee (IEC) or waiver.

\section{Participating study sites}

The study was conducted at eight independent International Standard Organization (ISO)-certified clinical laboratories (Table 1) in Berlin, Germany (Medizinisches Infektiologiezentrum Berlin); Aachen, Germany (PZB Aachen); Padua, Italy (Padua University Hospital, Padova); Madrid, Spain (Hospital Universitario Ramón y Cajal); El Prat de Llobregat, Spain (Laboratori de Referencia de Catalunya); Glasgow, UK (West of Scotland Specialist Virology Centre); Creteil, France (Hospital Universitaire Henri Mondor); and Johannesburg, South Africa (Lancet Laboratories). The clinical laboratories comprised a mix of hospital-based and privately-owned entities.

\section{Analyzers}

At each study site, one Alinity m analyzer was used to compare the workflow against multiple routine analyzers. The routine analyzers included in the study and their key features are summarized in Table 1 and Supplemental Table S1, respectively. The Alinity m (Abbott Molecular, Des Plaines, IL, USA) is a fully automated, continuous, random-access molecular diagnostic analyzer using real-time PCR and ReadiFlex ${ }^{\circledR}$ technology [12]. It is a floor analyzer with a processing capacity of 300 samples in approximately $8 \mathrm{~h}$, and has an amplification reagent capacity of 20 reagent packs, which can be stored onboard for 30 days. The time to first result is $<2 \mathrm{~h}$ and the ReadiFlex technology enables the system to run statum (STAT) samples.

\section{Samples and testing procedures}

Clinical samples were received at each laboratory in accordance with their established criteria for respective sample types over a period of 5-15 days. Clinical specimens for the requested analyte were tested on the routine analyzers and held at either $4-8{ }^{\circ} \mathrm{C}$ or frozen at $-20^{\circ} \mathrm{C} /$ $-70^{\circ} \mathrm{C}$ for long-term storage. Requested tests and the date and time of sample arrival, sample onboarding, sample aspiration, and result reporting were documented. Batch testing on routine analyzers required clinical samples to be sorted, stored, and frozen until 


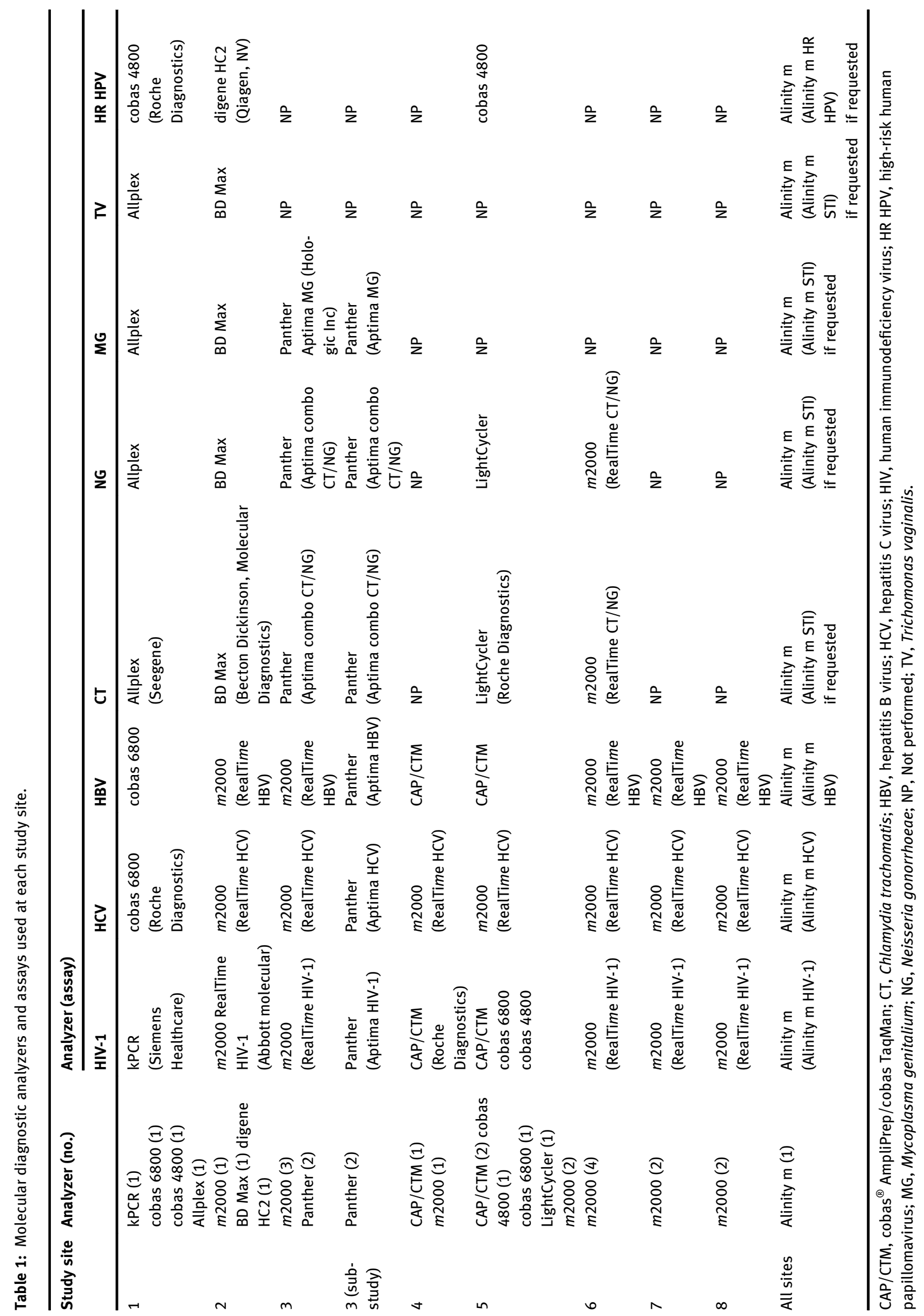


sufficient numbers of samples were collected to maximize reagent usage and minimize waste. Alinity m provides random-access capabilities; therefore, sorting, storing, freezing, and thawing procedures were not required. Assays were run per each laboratory's testing SOP and performed per the manufacturers' package insert instructions. Testing on the Alinity m instrument was conducted in accordance with the Alinity m System Operations Manual, assay-specific application files, assay-specific instructions for use, and the study protocol.

The Alinity m workflow at six sites used parallel water samples to simulate sample arrival in the laboratory. At study site 3, consecutive clinical samples received by the laboratory were split into two aliquots. The first aliquot was tested with the routine analyzer per laboratory procedures while the second aliquot was tested immediately on the Alinity m analyzer. At study site 6 , consecutive clinical samples received by the laboratory were first processed according to the routine workflow. To simulate a routine workflow on Alinity m, samples of a specific testing day were de-identified and processed on Alinity $\mathrm{m}$ based on their documented arrival times. Additionally, at three study sites, priority samples were received (or arbitrarily defined) and tested as STAT samples on the Alinity m platform and, if available, on the comparator routine analyzer to evaluate the impact of STAT requests on workflow metrics.

\section{Workflow metrics}

Three time periods (Figure 1), reflecting the major aspects of workflow in the clinical laboratory, were measured for the routine analyzers and Alinity m: (i) "Total TAT" was defined as the time from arrival of samples into the molecular diagnostic laboratory to result reporting by the analyzer; this period included all pre-analytical procedures (e.g., centrifugation, storing, sorting, freezing, and thawing to batch samples, if applicable); (ii) "Sample onboard TAT" was defined as the time period from sample loading and automated or manual start of testing (depending on the analyzer) to result reporting by the analyzer; and (iii) "Processing TAT" was defined as the time period from sample aspiration by the analyzer to result reporting by the analyzer; this measure focused on analyzer performance and was only assessed for Alinity m.

\section{Evaluation of TATs}

Total TAT and sample onboard TAT using the standard workflows on multiple routine analyzers (study sites 1-5) or on the batch workflow analyzer $m 2000$ (study sites 6-8) were compared to those of Alinity m. Sample onboard TAT of STAT samples was assessed for Alinity $m$ at three study sites and compared to the Panther (Hologic, Inc. Massachusetts) system at one study site. Evaluation of processing TAT on Alinity m was conducted "AT" all study sites.

Study site 3 conducted an additional evaluation of TATs comparing two Panther analyzers to a single Alinity m analyzer using fresh clinical specimens over a period of two days. Since each Panther analyzer can run a maximum of four different assays, the site compared two Panther analyzers to one Alinity $\mathrm{m}$ analyzer to accommodate continuous testing of the six analytes per study protocol. Panther 1 was dedicated to running the Aptima CT/NG and Aptima MG assays. Panther 2 was dedicated to running the Aptima HIV-1, HCV, and HBV assays. Analyzer-specific racks were loaded onto the analyzers simultaneously. Result reporting times from the analyzers were recorded for all samples.

\section{Results}

\section{Total TATs for routine analyzers and Alinity $m$ (Abbott)}

We examined the total TATs (i.e., from sample arrival at the laboratory to result reporting) for standard workflows of multiple clinical routine analyzers compared to Alinity $\mathrm{m}$ (Table 2 ). Total TATs for Alinity $\mathrm{m}$ ranged from 5 to $29 \mathrm{~h}$ for $100 \%$ of results, compared to standard workflows on routine analyzers, which ranged from 4 to 21 days (Table 2 and Supplemental Figure S1). A period of 21 days for total TAT was observed at study site 2; this result was a function of their routine workflow for HPV diagnosis, which includes batching every 10 days as only a few samples are analyzed with the digene HC2 HPV DNA (Qiagen, NV) test. Total TATs for routine analyzers varied by requested assay (Figure 2A, C, E, G, I, $\mathrm{K}, \mathrm{M}, \mathrm{O}$ ), whereas total TATs for Alinity $\mathrm{m}$ were consistent, rapid, and independent of the requested assay (Figure 2B, D, F, H, J, L, N, P).

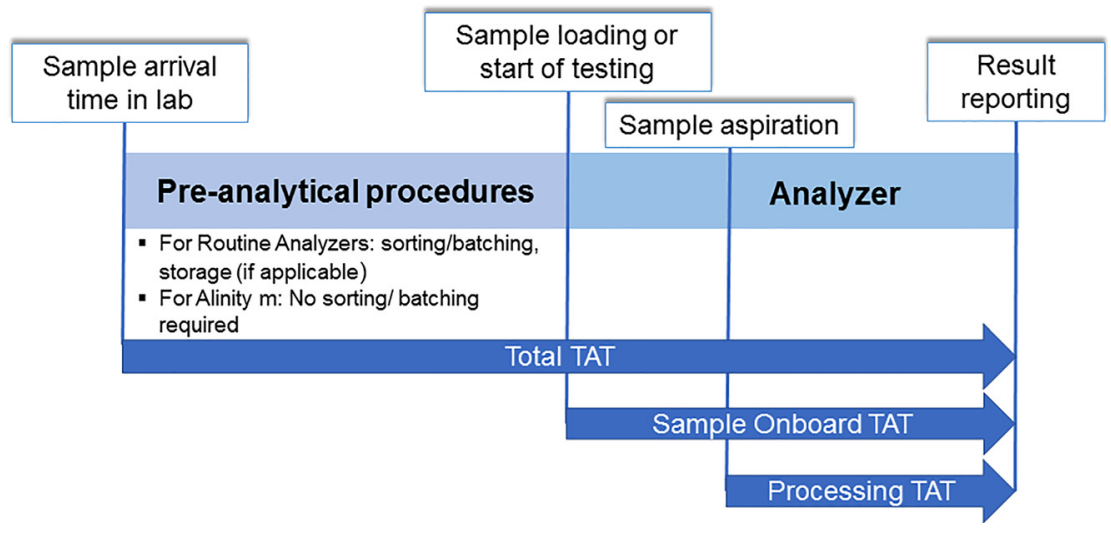

Figure 1: Workflow metrics for the various aspects in the clinical laboratory. Three turnaround time (TAT) periods were measured for routine analyzers and Alinity $\mathrm{m}$. The processing TAT metric was only measured for Alinity $\mathrm{m}$. 


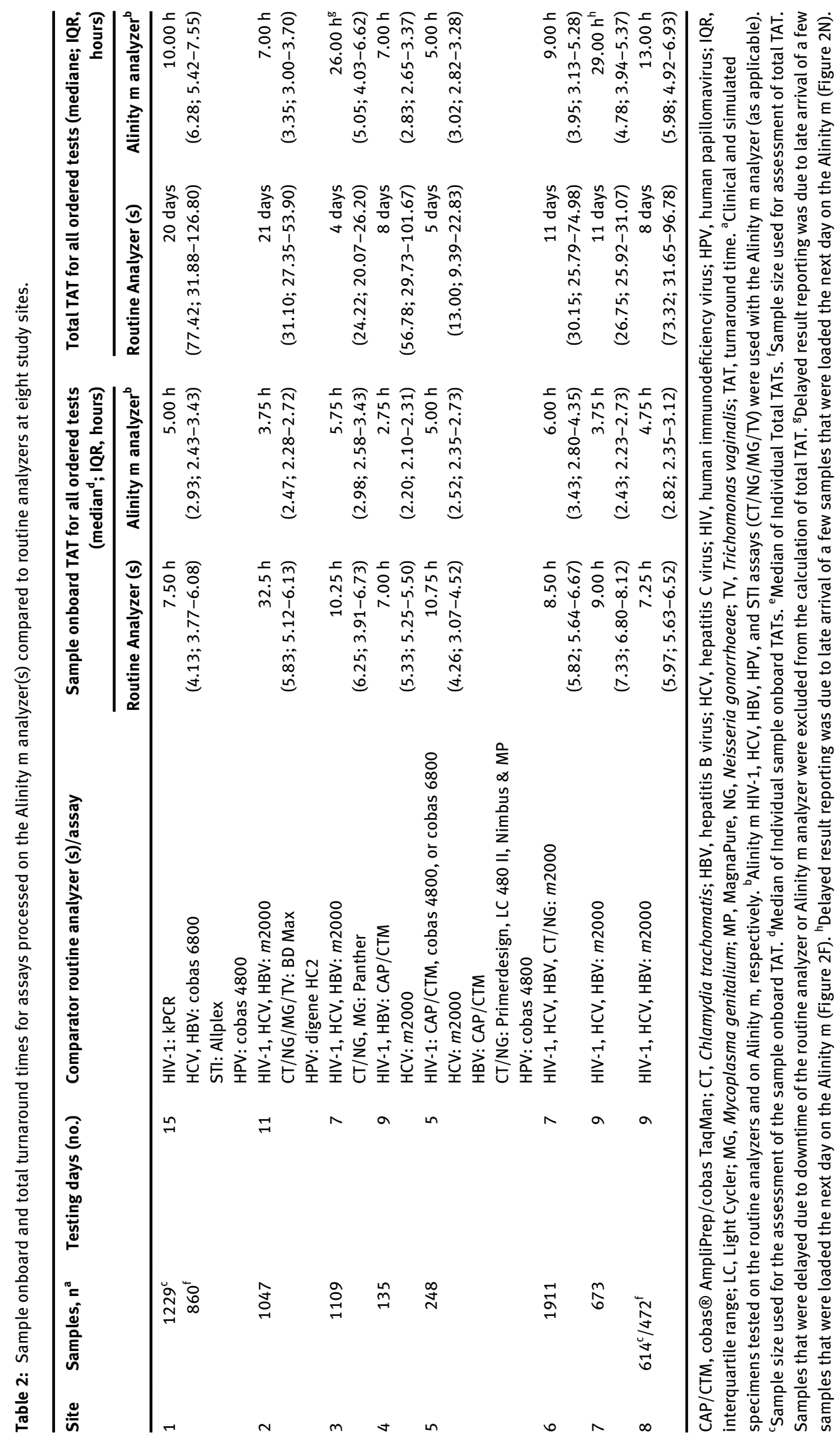



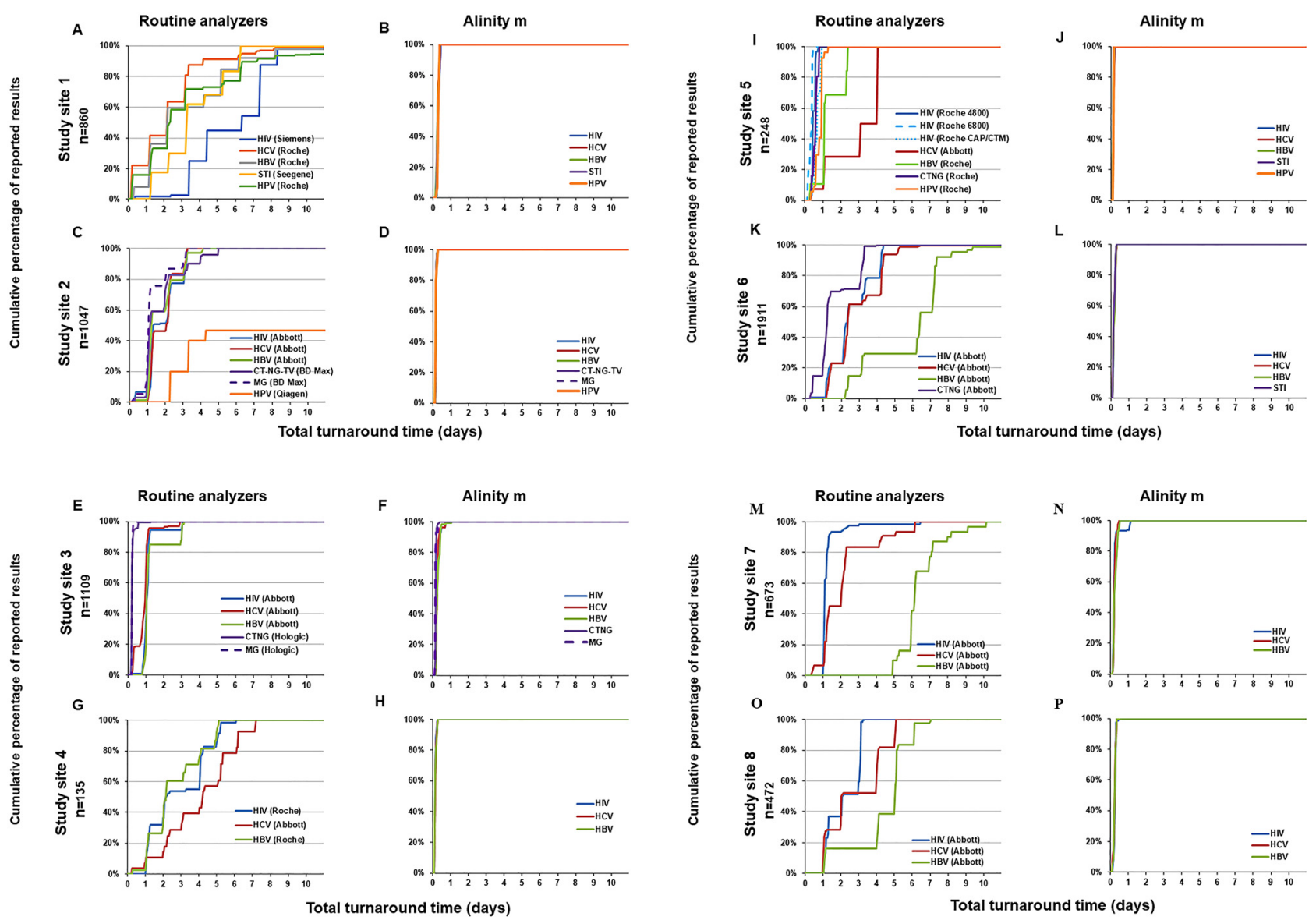

Figure 2: Total turnaround time (TAT) with the consolidated, continuous, random-access workflow of Alinity m analyzer compared to the standard workflows of multiple routine analyzers.

Total TAT was defined as the time from sample arrival in the laboratory until reporting of results. Data are presented as time to completion for the cumulative percentage of samples. Left panels: total TATs broken out by assay for the routine analyzers. Right panels: total TATs broken out by assay for the Alinity $m$ analyzer (HIV is shown in blue; HCV, red; HBV, green; STI, purple; and HPV, orange). Study site 1 (A, B; $n=860$ ); study site 2 (C, D; $n=1047 ;$ STI: CT/NG/TV [solid line], MG [dashed line]); study site 3 (E, F; $n=1,109 ; S T I: ~ C T / N G$ [solid line], MG [dashed line]); study site 4 (G, H; $n=135$ ), study site 5 (I, J; $n=248$; HIV: cobas 4800 [solid line], cobas 6800 [dashed line], CAP/CTM [dotted line]), study site 6 (K, L; $n=1,911)$, study site $7(M, N ; n=673)$, and study site $8(0, P ; n=472)$.

\section{Sample onboard TATs for multiple routine analyzers compared to Alinity $m$}

In evaluating sample onboard TAT, routine analyzers used at each study site reported results for all ordered tests with overall sample onboard TATs ranging from 7.00 to $32.50 \mathrm{~h}$ across testing sites 1-5 (medians of individual sample onboard TAT ranged from 4.13 to $6.25 \mathrm{~h}$; Table 2). Sample onboard TATs were dependent on the number of samples, tested analyte, and analyzer used (Figure 3A, C, E, G, I). The Alinity $\mathrm{m}$ analyzer at each site reported results for all tests ordered with overall sample onboard TATs ranging from 2.75 to $6 \mathrm{~h}$ (medians of individual sample onboard TATs ranged from 2.20 to $2.98 \mathrm{~h}$ ) in a continuous manner, independent of the analyte tested (Figure 3B, D, F, H, J).

\section{Sample onboard TATs for the $\mathrm{m} 2000$ analyzer and Alinity $\mathrm{m}$}

The $m 2000$ (a batch analyzer) at testing sites 6-8 reported results for all ordered tests with overall sample onboard TATs between 7.25 and $9.00 \mathrm{~h}$ after samples were loaded (medians of individual sample onboard TATs ranged from 5.82 to $7.33 \mathrm{~h}$; Table 2 and Figure 3K, M, O), whereas Alinity $\mathrm{m}$ (a continuous, random-access analyzer) completed result reporting for all ordered tests between 3.75 and $5.75 \mathrm{~h}$ after samples were loaded (medians of individual sample onboard TATs ranged from 2.43 to $3.43 \mathrm{~h}$ ). The sample onboard TATs on Alinity m were independent of the tested analyte (Table 2 and Figure 3L, $\mathrm{N}, \mathrm{P})$. 

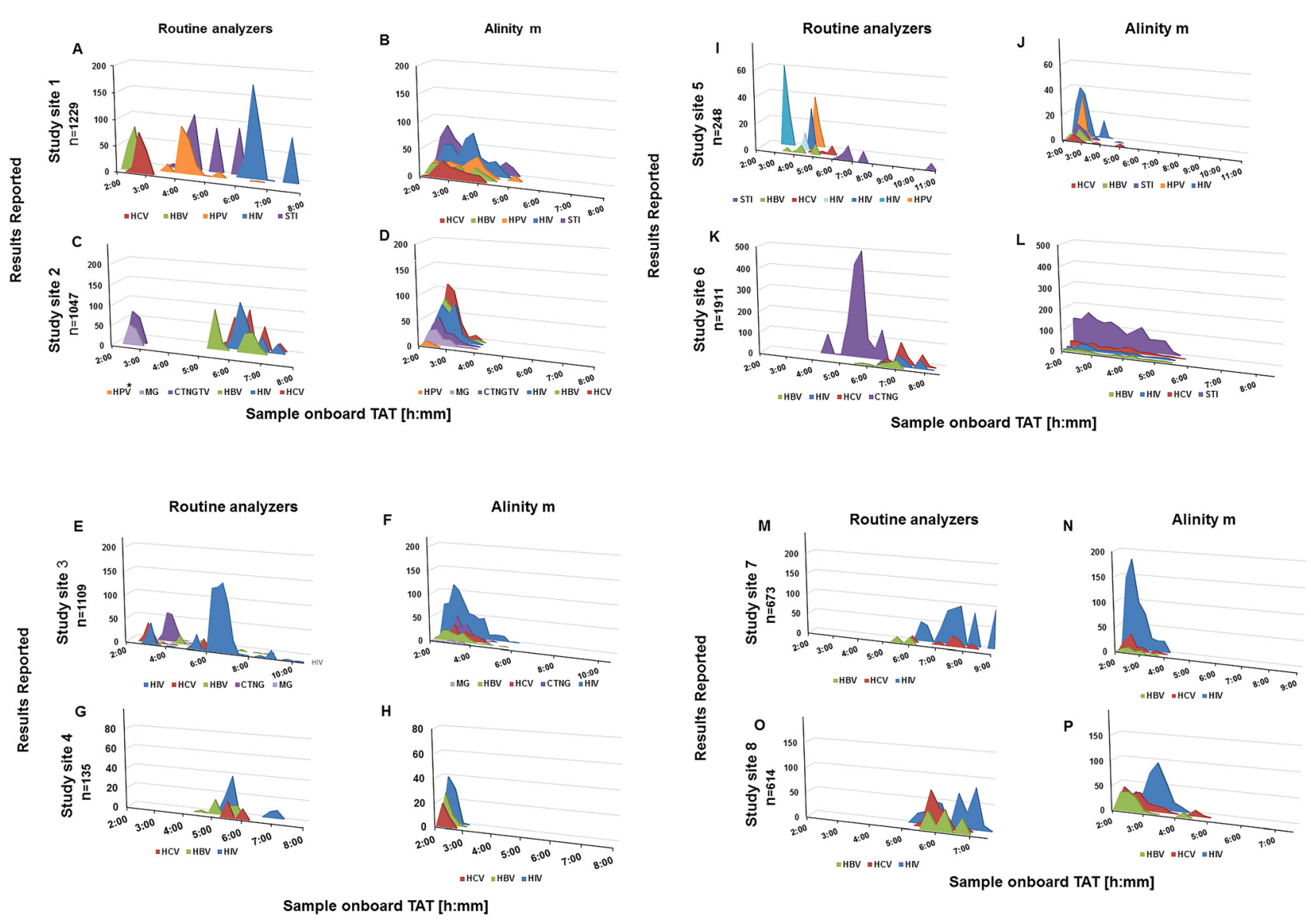

Figure 3: Comparison of sample onboard turnaround time (TATs) with multiple routine analyzers vs. the consolidated workflow of the Alinity $\mathrm{m}$ analyzer.

The time from sample onboarding to result reporting on the Alinity $m$ analyzer compared to the time from the start of processing to result reporting with multiple routine analyzers at eight study sites for the various assays shown: study site 1 ( $A, B ; n=1,229$ ); study site 2 (C, D; $n=1,047 ;{ }^{*} 15$ additional HPV results were obtained after 32 h); study site 3 (Phase 1; E, F; n=1,109); study site 4 (G, H; $n=135$ ); study site 5 (I, J; $n=248)$; study site 6 (K, L; $n=1911)$; study site 7 (M, N; $n=673)$; and study site $8(0, P ; n=614)$.

\section{Sample onboard TATs for Panther analyzers and Alinity $m$}

At study site 3, TATs from sample onboarding to result reporting for various tests requested using the routine workflow were compared for two Panther analyzers and one Alinity $\mathrm{m}$ analyzer. On day 1,180 samples were processed utilizing the two Panther analyzers in $8.68 \mathrm{~h}$ compared to $8.00 \mathrm{~h}$ for Alinity $\mathrm{m}$ (Figure 4). On day 2, an analysis of an additional 169 samples was completed in $7.13 \mathrm{~h}$ on the two Panther analyzers, compared to $6.33 \mathrm{~h}$ on Alinity m (Supplemental Figure S2).

\section{Alinity $m$ processing TATs}

To further explore the consistency of result reporting of Alinity $\mathrm{m}$ in a random-access testing mode, we compared processing TATs on Alinity $m$ analyzers across the eight laboratories $(n=7,476)$. All tests ordered on Alinity $\mathrm{m}$ had results reported within a range from 113 to $117 \mathrm{~min}$, with 95\% of all ordered tests reported in 115 min (Figure 5). Processing TATs were independent of the assay combination and were consistent across the eight laboratories. None of the comparator routine analyzers were capable of simultaneously processing the above assay combination $[11,13]$.

\section{Alinity m STAT sample processing}

A total of 3,754 samples were tested across three study sites; of these, $3.4 \%$ of samples $(n=126)$ were ordered as STAT samples. The mean sample onboard TAT for STAT samples on Alinity m was $2.36 \mathrm{~h}( \pm 0.19 \mathrm{~h}$ standard deviation). The sample onboard TATs of routine samples loaded 


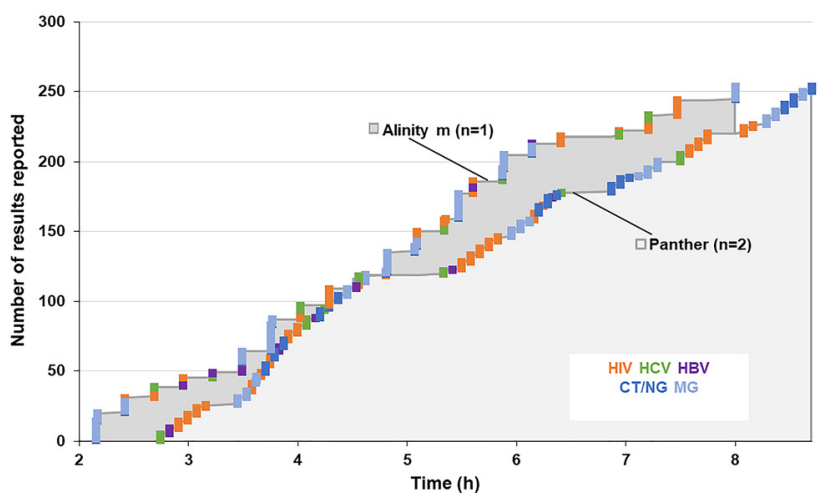

Figure 4: Time to result with two random-access Panther analyzers compared to one Alinity $m$ analyzer with ReadiFlex technology testing the same workload (study site 3 , day $1, n=180$ samples). "Number of results" is the order in which results were generated by each system. The lighter shaded area represents a combination of tests on the two Panther analyzers, and the darker shaded area represents the tests run on Alinity m. Colored blocks represent the tests run on the different analyzers.

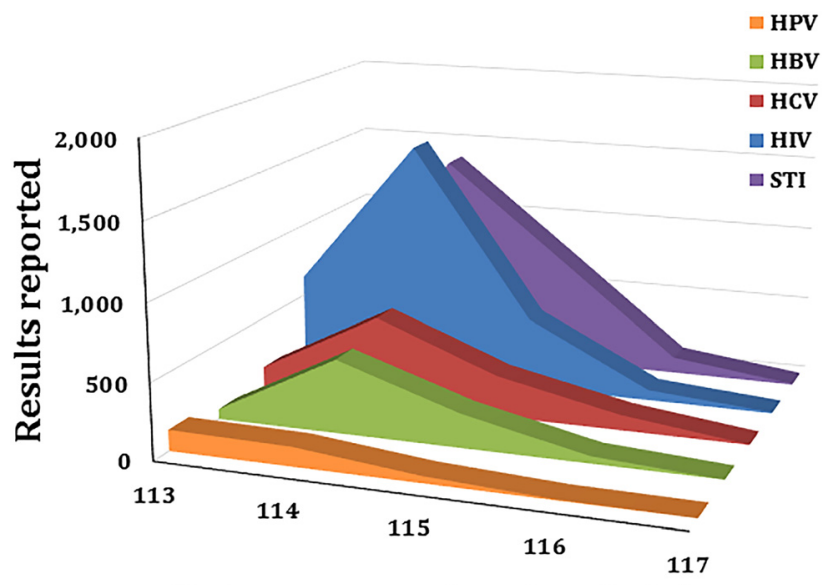

Alinity m Processing Time [min]

Figure 5: Processing turnaround time (TAT) for Alinity $m$ per assay. Processing TAT, defined as the time from sample aspiration until result reporting, was determined for a total of 7,476 samples run on Alinity $\mathrm{m}$ analyzers across all eight study sites.

on Alinity m were not affected by prioritizing STAT samples (Figure 6).

In a direct comparison at one site, the sample onboard TATs for three STAT STI samples tested on Alinity m were $2.48,2.33$, and $2.48 \mathrm{~h}$, compared to $4.28,4.85$, and $4.37 \mathrm{~h}$ on the Panther analyzer. A STAT HIV-1 sample had a sample onboard TAT of $3.28 \mathrm{~h}$ on Alinity m compared to $3.78 \mathrm{~h}$ on Panther.

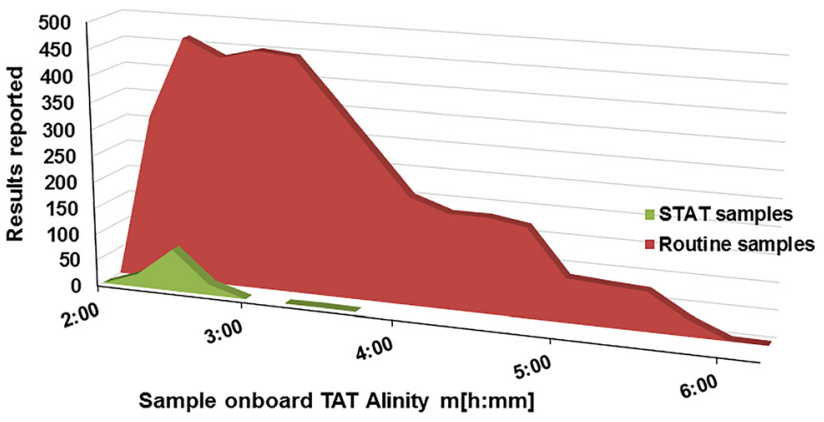

Figure 6: Comparison of routine vs. STAT sample onboard turnaround time (TATs) on Alinity $\mathrm{m}$.

A total of 3,754 samples including 3.4\% STAT samples $(n=126)$ were tested across three study sites. STAT samples are indicated in green.

\section{Discussion}

This study is the first evaluation of the impact of Alinity $m$ (Abbott) on workflow in a clinical laboratory setting. Our study showed that consolidating HIV-1, HCV, HBV, HR HPV, and STI testing onto a single analyzer and transitioning to daily testing would enable laboratories to achieve significant improvements in workflow and operations. The total TATs for combinations of 3-5 assays on Alinity $\mathrm{m}$ were consistently shorter than those for the comparator routine analyzers, independent of assays tested and study sites. The study was conducted across eight different clinical laboratories that use various analyzers ranging from random-access to batch workflows to obtain functional data reflecting the breadth of molecular laboratories and operational workflows [14-17]. A comparison of clinical performance was beyond the scope of the current study, which focused exclusively on the workflow metrics by utilizing simulated or residual specimens. Clinical performance data between Alinity $\mathrm{m}$ and various molecular assays is published elsewhere [18-22]. In these studies, the Alinity m HIV-1, HCV, HBV, HR HPV assays were found to be accurate and reproducible with high agreement to comparator assays. Potential discordant quantitation between any two methodologies could be due to sample storage length or temperature between the testing times of the two assays and intrinsic differences in the assay design features (e.g., standardization, calibration strategy, target regions) [23, 24].

The continuous, random-access workflow of the Alinity $m$ (Abbott) and Panther (Hologic) analyzers provided same-day sample results reporting, whereas the analyzers working in batches provided results over several days. Higher total TATs were observed for routine analyzers, which required batching samples and running selected 
assays to optimize reagent usage. Direct comparison of the Panther and Alinity $m$ analyzers demonstrated that a single Alinity $m$ required less time than two Panther analyzers to process the same number of samples. This finding could be attributed to specific characteristics of the Panther analyzer. For example, new assay kits cannot be loaded onto the Panther analyzer until all ordered samples utilizing the onboard assay kits are completed. System fluids and waste can only be emptied and replenished after all sample processing is completed. Additionally, the Panther analyzer requires at least three calibrations every $24 \mathrm{~h}$ in addition to three controls per assay. Finally, Alinity m was able to prioritize STAT samples without compromising the TAT for on board routine samples.

Hands-on time was measured at two sites by an independent workflow consultant specialized in diagnostic product research (Enterprise Analytics Corporation, Stamford, CT, USA). Hands-on time during routine use ranged from $6 \mathrm{~min}$ for Alinity $\mathrm{m}$ and Panther to $10 \mathrm{~min}$ for cobas 6800 (Roche Diagnostics), with a maximum of $0.5 \mathrm{~h}$ for the analyzers (data not shown). Semi-automated methods such as Versant kPCR (Siemens Healthcare) and Allplex (Seegene Inc, Seoul) required a hands-on time of approximately $60 \mathrm{~min}$ (data not shown). Manual handling of samples and reagents may not only increase the various workflow parameters measured, but may also increase the risk of contamination and handling errors [15]. A consistently shorter total TAT for results reporting, inclusive of all pre-analytic activities, obtained with the Alinity $m$ analyzer could offer operational benefits on overall workflow in the clinical laboratory that extends beyond analyzer performance. Furthermore, the use of different molecular analyzers in the same laboratory may yield an inefficient utilization of laboratory personnel and technological resources resulting in reduction of operating efficiencies.

Limitations of the study include the use of simulated samples at six of the eight study sites due to insufficient volume in the residual samples to test across multiple comparator analyzers and retrospective use of residual samples at another site to assess workflow TATs on Alinity $\mathrm{m}$. This approach simulated a routine workflow with Alinity $\mathrm{m}$, albeit in an orchestrated manner. The strengths of this study include its international, multicentric design and testing of clinical samples in a real-world setting, across various assays with multiple routine analyzers and standard workflows.

Our findings on the workflow impact of test consolidation with fully automated molecular platforms have clinical implications for molecular laboratories. The attributes of Alinity m (Abbott), utilizing ReadiFlex technology with assay-specific, lyophilized, unit-dose reagents, eliminate the need for sorting and batching, allowing for independent processing of any sample at any time. The ability to run STAT samples without disrupting routine sample processing provides rapid test results in situations where immediate clinical decisions must be made. Same-day reporting of test results can shorten the time between diagnosis and treatment with the potential for improvements in patient care and clinical outcomes.

Acknowledgments: Abbott Molecular Inc., Des Plaines, IL, USA and Abbott GmbH, Wiesbaden, Germany for proposing and promoting this work.

Research funding: The study was funded by Abbott Molecular Inc.

Author contributions: All authors have accepted responsibility for the entire content of this manuscript and approved its submission.

Competing interests: AV, AG, EG, FA, FO, GN, MK, RE, RG, and MP have no conflicts of interest. HK has received speaker honorariums and/or travel grants from AbbVie, GILEAD, Hexal, Hormosan, Janssen, MSD, Roche, and ViiV. JCG has received speaker honorariums from Hologic and Gilead, research grants from Roche and AbbVie, and travel grants from Siemens and Abbott. LMG has received a speaker honorarium from Abbott and a travel grant from Hologic. MO has received speaker honorariums from Roche, Hologic, Cepheid, and Siemens; received travel grants from Roche, Abbott, Hologic, and Vela Diagnostics; and acted as advisor for Cepheid, Siemens, and Abbott. PB has received speaker honorariums from Abbott, Cepheid, and Hologic; received travel grants from Abbott, Beckman Coulter, Cepheid, Hologic, and Roche; and acted as advisor for Abbott, Beckman Coulter, and Cepheid. SC has acted as advisor for Abbott and Cepheid. BR, JD, and KP are employees of Abbott GmbH. AMJ, DL, MJP, and NM are employees of Abbott Molecular Inc. EG has received an educational grant from Abbott.

Ethical approval: Residual patient specimens (where applicable) were sourced per site policies, procedures, and applicable local regulations, including approval by the site's Independent Ethics Committee (IEC) or waiver.

\section{References}

1. Fairfax MR, Bluth MH, Salimnia H. Diagnostic molecular microbiology: a 2018 snapshot. Clin Lab Med 2018;38:253-76.

2. Sloots TP, Nissen MD, Ginn AN, Iredell JR. Rapid identification of pathogens using molecular techniques. Pathology 2015;47:191-8. 
3. Scagnolari C, Turriziani O, Monteleone K, Pierangeli A, Antonelli $\mathrm{G}$. Consolidation of molecular testing in clinical virology. Expert Rev Anti Infect Ther 2017;15:387-400.

4. Caliendo AM, Gilbert DN, Ginocchio CC, Hanson KE, May L, Quinn TC, et al. Better tests, better care: improved diagnostics for infectious diseases. Clin Infect Dis 2013;57 (3 Suppl):S139-70.

5. Workowski KA, Bolan GA, Centers for Disease C, Prevention. Sexually transmitted diseases treatment guidelines. 2015. MMWR Recomm Rep 2015;64:1-137.

6. Nolte FS. Counterpoint: distributed model for molecular diagnostics. Clin Chem 2019;66:140-2.

7. Greub G, Sahli R, Brouillet R, Jaton K. Ten years of R\&D and full automation in molecular diagnosis. Future Microbiol 2016;11: 403-25.

8. Cobb B, Simon CO, Stramer SL, Body B, Michell PS, Reisch N, et al. The cobas ${ }^{\circledR} 6800 / 8800$ System: a new era of automation in molecular diagnostics. Expert Rev Mol Diagn 2017;17: 167-80.

9. Frontzek A, Aretzweiler G, Winkens D, Duncan D, Marlowe EM. High-volume workflow and performance comparisons for Chlamydia trachomatis and Neisseria gonorrhoeae testing using automated molecular platforms. BMC Infect Dis 2019;19:797.

10. Hawkins RC. Laboratory turnaround time. Clin Biochem Rev 2007; 28:179-94. https://www.ncbi.nlm.nih.gov/pmc/articles/ PMC2282400/pdf/cbr28_4p179.pdf.

11. Abbott Molecular. Alinity $m$ system operations manual. Des Plaines, IL: Abbott Molecular Inc.; 2019.

12. Abbott Molecular Inc. Reshaping the Future of Molecular Diagnostic Testing: the Alinity $m$ System. AMD; 2019:14164 p.

13. CAP TODAY. Automated molecular platforms. [updated October 2018]. Available from: http://digital.olivesoftware.com/Olive/ ODN/CAPTODAY/ [Accessed 24 Mar 2020].

14. Dize L, West S, Williams JA, Van Der Pol B, Quinn TC, Gaydos CA. Comparison of the Abbott m2000 RealTime CT assay and the Cepheid GeneXpert CT/NG assay to the Roche Amplicor CT assay for detection of Chlamydia trachomatis in ocular samples from Tanzania. J Clin Microbiol 2013;51:1611-3.

15. Ratnam S, Jang D, Gilchrist J, Smieja M, Poirier A, Hatchette T, et al. Workflow and maintenance characteristics of five automated laboratory instruments for the diagnosis of sexually transmitted infections. J Clin Microbiol 2014;52:2299-304.
16. Scott LE, Noble LD, Moloi J, Erasmus L, Venter WD, Stevens W. Evaluation of the Abbott m2000 RealTime human immunodeficiency virus type 1 (HIV-1) assay for HIV load monitoring in South Africa compared to the Roche Cobas AmpliPrep-Cobas Amplicor, Roche Cobas AmpliPrep-Cobas TaqMan HIV-1, and BioMerieux NucliSENS EasyQ HIV-1 assays. J Clin Microbiol 2009;47:2209-17.

17. Troppan KT, Stelzl E, Violan D, Winkler M, Kessler HH. Evaluation of the new VERSANT HIV-1 RNA 1.0 Assay (KPCR) for quantitative detection of human immunodeficiency virus type 1 RNA. I Clin Virol 2009;46:69-74.

18. Bonanzinga S, Onelia F, Jackson K, Glass A, Maree L, Krügel M, et al. Multicenter clinical evaluation of Alinity $\mathrm{m} \mathrm{HBV}$ assay performance. J Clin Virol 2020;129:104514.

19. Chevaliez S, Onelia F, Pacenti M, Goldstein E, Galan JC, MartinezGarcia L, et al. Multicenter clinical evaluation of Alinity m HCV assay performance. J Clin Virol 2020;129:104531.

20. Braun P, Glass A, Maree L, Krügel M, Pacenti M, Onelia F, et al. Multicenter clinical comparative evaluation of Alinity m HIV-1 assay performance. J Clin Virol 2020;129:104530.

21. Maree L, Krügel M, Reinhardt B, Glass A. Evaluation of the Alinity $m$ HIV-1 assay for the quantification of HIV-1 RNA plasma viral load in a high-throughput molecular laboratory in South Africa. J Clin Virol 2020;132:104644.

22. Oštrbenk Valenčaka A, Šterbenca A, Seme K, Poljak M. Alinity $m$ HR HPV assay fulfils criteria for human papillomavirus test requirements in cervical cancer screening settings.

J Clin Microbiol 2019;58. https://doi.org/10.1128/ JCM.01120-19.

23. Mouna L, Pallier C, Proust S, Pregermain C, Roque-Afonso AM. Comparison of the Abbott Alinity $\mathrm{m}$ and $\mathrm{m} 2000$ assays for the quantification of HIV-1, HCV and HBV in clinical samples. J Clin Virol 2020;126:104331.

24. May S, Adamska E, Tang J.W. Evaluating the aptima HIV-1 quant Dx, HCV quantDx and HBV quant assays against Abbott HIV-1, HCV and HBV Realtime assays. J Clin Virol 2018;106:7-10.

Supplementary Material: The online version of this article offers supplementary material (https://doi.org/10.1515/labmed-20200102). 